\title{
Weakly nonlinear analysis of localized bulging of an inflated hyperelastic tube of arbitrary wall thickness
}

\author{
Yang Ye ${ }^{\mathrm{a}}$, Yang Liu ${ }^{\mathrm{a}}$, Yibin $\mathrm{Fu}^{\mathrm{b}, *}$ \\ ${ }^{a}$ Department of Mechanics, Tianjin University, Tianjin 300072, China \\ ${ }^{b}$ School of Computing and Mathematics, Keele University, Staffordshire ST5 5BG, UK
}

\begin{abstract}
A weakly nonlinear analysis is conducted for localized bulging of an inflated hyperelastic cylindrical tube of arbitrary wall thickness. Analytical expressions are obtained for the coefficients in the amplitude equation despite the fact that the primary deformation is inhomogeneous and the incremental governing equations have variable coefficients. It is shown that for each value of wall thickness a localized bulging solution does indeed bifurcate subcritically from the primary solution for almost all values of fixed axial force or fixed axial stretch for which the bifurcation condition is satisfied, as reported in all previous experimental studies, but there also exist extreme cases of fixed axial stretch for which localized bulging gives way to localized necking. Validation is carried out by comparing with results obtained under the membrane assumption and with fully numerical simulations based on Abaqus. It is shown that even for thin-walled tubes the membrane approximation becomes poorer and poorer as the tube is subjected to increasingly larger and larger axial stretch or force prior to inflation.
\end{abstract}

Keywords: Localized bulging, rubber tubes, necking, bifurcation, nonlinear elasticity.

\section{Introduction}

This paper continues our systematic studies on localized bulging of inflated hyperelastic tubes for the entire range of wall thickness. Based on the membrane assumption, our early studies include the establishment of localized bulging as a bifurcation phenomenon (Fu et al., 2008), and analysis of imperfection sensitivity (Fu \& Xie, 2012) and stability of the bulged configurations (Ilíchev \& Fu, 2012, 2014; Fu \& Xie, 2010, 2012; Fu \& Ilíchev, 2015). Our recent studies have abandoned the membrane assumption and attention has turned to tubes of arbitrary wall thickness. It was shown in Fu et al. (2016) that the bifurcation condition for localized bulging of inflated tubes of arbitrary wall thickness takes a very simple form, namely that the Jacobian determinant of the internal pressure and resultant axial force as

\footnotetext{
${ }^{*}$ Corresponding author at: School of Computing and Mathematics, Keele University, Staffordshire ST5 $5 \mathrm{BG}, \mathrm{UK}$

Email address: y.fu@keele.ac.uk (Yibin Fu)
} 
functions of two principal stretches is zero. This result makes it possible to examine the effect of rotation (Wang et al., 2017b), fibre-reinforcement (Wang \& Fu, 2018), and multi-layering (Liu et al., 2019; Ye et al., 2019) in a systematic manner. An experimental study guided by these newly emerged theoretical results has also been conducted (Wang et al., 2019).

When the membrane assumption is used, the governing equations are ordinary differential equations and due to the existence of two conservation laws the entire inflation process can be understood analytically or semi-analytically (Pearce \& Fu, 2010). When the membrane assumption is removed and tubes of arbitrary wall-thickness are considered, the governing equations are nonlinear partial differential equations, and as a result even a weakly nonlinear near-critical analysis becomes a non-trivial task. Currently, the only means to understand the post-bifurcation behaviour is through numerical simulations. The current paper makes the first analytical step. The main question to be addressed is whether localized bulging can indeed take place when the bifurcation condition is satisfied and, if it can, whether it is always a sub-critical bifurcation for all values of wall-thickness. This is achieved through derivation of an explicit weakly nonlinear amplitude equation.

Our previous and current studies draw together three different strands of research on circular cylindrical hyperelastic tubes that are subject to large deformations. The first strand consists of bifurcation and stability studies in which the bifurcation or instability modes are periodic in the axial and/or azimuthal direction; see, for instance, Shield (1972), Haughton \& Ogden (1979a,b), Chen (1997), Chen \& Haughton (2003), Merodio \& Haughton (2010), and Rodriguez \& Merodio (2011). The second strand is concerned with the socalled "limiting-point" instability, which refers to the fact that under uniform inflation the pressure as a function of internal volume may reach a maximum; see Alexander (1971), Benedict et al. (1979), Kanner \& Horgan (2007), Ren et al. (2011), Horny et al. (2015), and the references therein. The third strand comprises analytical, experimental and numerical studies of localized bulging; see, for instance, Yin (1977), Chater \& Hutchinson (1991), Kyriakides \& Chang (1990, 1991), Shi \& Moita (1996), Pamplona et al. (2006), Goncalves et al. (2008). It is now understood that (i) the pressure maximum in the limiting-point stability analysis is the initiation pressure for localized bulging when the resultant axial force is fixed, but this correspondence may not exist when other end conditions are considered (for instance, the case of fixed axial length), and (ii) the bifurcation condition of Haughton \& Ogden (1979a) with the mode number in the axial direction set to zero is in fact also the bifurcation condition for localized bulging although the mode corresponds to an extra uniform expansion in the radial direction and is seemingly unrelated to localized bulging.

Inflation of a circular cylindrical tube is a fundamental problem in finite elasticity (Green \& Zerna, 1954). The uniform inflation solution can be obtained in closed-form even for the most general strain-energy function and can be used as a bench-mark solution in many applications. It is one of the simplest situations to derive the Kortewed-de Vries equation and hence to demonstrate the existence of solitary waves under the combined effects of 
nonlinearity and weak dispersion (Fu \& Ilíchev, 2010). It is also probably the simplest prototypical example to demonstrate the coexistence of two uniform deformations and how a high-gradient theory in lower dimension can be derived from the exact nonlinear elasticity theory (Lestringant \& Audoly, 2018). Recent interest in the localized bulging problem has also been spurred by its relevance to modelling aneurysm initiation and rupture (Ren, 2007; Fu et al., 2012; Varatharajan \& DasGupta, 2017; Alhayani et al., 2013, 2014; Demirkoparan \& Merodio, 2017b), suppression of localized bulging in energy harvesting devices (Bucchi \& Hearn, 2013a,b), and potential use of electroelastic tubes in actuators ( $\mathrm{Lu}$ et al., 2015; An et al., 2015; Wang et al., 2017a). Other recent studies have addressed the effects of swelling (Demirkoparan \& Merodio, 2017a), viscoelasticity/chemorheology (Wineman, 2015, 2017), and plasticity (Takla, 2018).

Post-buckling analysis was routinely carried out for solids and structures described by approximate plate and shell theories (Hutchinson \& Koiter, 1972; Potier-Ferry, 1987), but in the context of nonlinear elasticity it is relatively limited; here stability and bifurcation analysis has predominantly been linear. The first nonlinear study seems to be the one by Sawyers \& Rivlin (1982), followed much later by Fu \& Rogerson (1994), Fu (1995), Fu \& Ogden (1999), and Triantafyllidis et al. (2007) which are all concerned with hyperelastic plates. The recent study by Chakrabarti et al. (2018) is also about a plate but is concerned with buckling patterns produced by the elastic Rayleigh-Taylor instability when a very soft plate is hanging below a rigid horizontal plane. The slightly more challenging geometry of a coated hyperelastic half-space was examined by Cai \& Fu (1999) and Hutchinson (2012); they showed that depending on how stiff the coating layer is relative to the half-space, the bifurcation can be super-critical or sub-critical. This result helps us understand why creases appear when the coating layer and half-space have comparable stiffness whereas robust sinusoidal patterns appear when the coating layer is much stiffer than the half-space (Cao \& Hutchinson, 2012). Weakly nonlinear analysis involving circular cylindrical geometry has recently been conducted by Richard et al. (2018) for the buckling of a spinning elastic cylinder and by Jin et al. (2019) for pattern formation in growing tubular tissues. Our current study also involves circular cylindrical geometry, but our analysis is concerned with a localized bifurcation mode. For explanations of the general methods of buckling/stability analysis, we refer to van der Heijden (2009) and Fu (2001).

The rest of this paper is divided into four sections as follows. In the next section we summarize previously known results for the primary deformation and the bifurcation condition, and derive the incremental equations. This is then followed in Section 3 by a derivation of the amplitude equation governing the shape of the localized bulging solution. In Section 4 we present numerical results and compare them with the membrane theory and Abaqus simulations. The paper is concluded with a summary of our main results and some additional discussions. 


\section{Problem formulation}

\subsection{Primary deformation}

Consider a sufficiently long circular cylindrical tube that is made of an incompressible hyperelastic material. It initially has inner radius $A$ and outer radius $B$, and when it is uniformly stretched in the axial direction and inflated by an internal pressure $P$, the inner and outer radii become $a$ and $b$, respectively. In terms of cylindrical polar coordinates, the deformation is given by

$$
r=r(R), \quad \theta=\Theta, \quad z=\lambda_{z} Z
$$

where $(R, \Theta, Z)$ and $(r, \theta, z)$ are the coordinates in the undeformed and deformed configurations, respectively, and $\lambda_{z}$ is the constant stretch in the axial direction. The deformation gradient $F$ is then given by

$$
F=\frac{r}{R} \boldsymbol{e}_{\theta} \otimes \boldsymbol{e}_{\theta}+\lambda_{z} \boldsymbol{e}_{z} \otimes \boldsymbol{e}_{z}+r^{\prime}(R) \boldsymbol{e}_{r} \otimes \boldsymbol{e}_{r},
$$

so that the three principal stretches are simply

$$
\lambda_{1}=\frac{r}{R}, \quad \lambda_{2}=\lambda_{z}, \quad \lambda_{3}=1 /\left(\lambda_{1} \lambda_{2}\right),
$$

where $\left(\boldsymbol{e}_{r}, \boldsymbol{e}_{\theta}, \boldsymbol{e}_{z}\right)$ is the common orthonormal basis for the two sets of cylindrical polar coordinates, and as in Haughton \& Ogden (1979b) we have identified the indices 1,2,3 with the $\theta-, z-$, and $r$-directions, respectively.

Incompressibility implies that

$$
r^{2}=\lambda_{z}^{-1}\left(R^{2}-A^{2}\right)+a^{2},
$$

which defines function $r(R)$ (or $R$ as a function of $r$ ). The constitutive behaviour is determined by the strain-energy function $W\left(\lambda_{1}, \lambda_{2}, \lambda_{3}\right)$, in terms of which the non-zero Cauchy stress components are given by

$$
\sigma_{i i}=\lambda_{i} \frac{\partial W}{\partial \lambda_{i}}-p, \quad \text { no summation on } i
$$

where $p$ is a Lagrangian multiplier associated with the constraint of incompressibility. It can then be shown (Haughton \& Ogden, 1979b) that in terms of a reduced strain-energy function $w\left(\lambda_{1}, \lambda_{2}\right)$ defined by

$$
w\left(\lambda_{1}, \lambda_{2}\right)=W\left(\lambda_{1}, \lambda_{2}, \lambda_{1}^{-1} \lambda_{2}^{-1}\right),
$$

the internal pressure $P$ and the resultant axial force $N$ are given by

$$
\begin{gathered}
P \equiv-\left.\sigma_{33}\right|_{r=a}=\int_{\lambda_{b}}^{\lambda_{a}} \frac{w_{1}}{\lambda^{2} \lambda_{z}-1} d \lambda \\
N \equiv 2 \pi \int_{a}^{b} \sigma_{22} r d r-\pi a^{2} P=\pi A^{2}\left(\lambda_{a}^{2} \lambda_{z}-1\right) \int_{\lambda_{b}}^{\lambda_{a}} \frac{2 \lambda_{z} w_{2}-\lambda w_{1}}{\left(\lambda^{2} \lambda_{z}-1\right)^{2}} \lambda d \lambda,
\end{gathered}
$$


where the two limits $\lambda_{a}$ and $\lambda_{b}$ are defined by

$$
\lambda_{a}=\frac{a}{A}, \quad \lambda_{b}=\frac{b}{B},
$$

and are related to each other through the incompressibility condition by

$$
\lambda_{a}^{2} \lambda_{z}-1=\frac{B^{2}}{A^{2}}\left(\lambda_{b}^{2} \lambda_{z}-1\right)
$$

With $\lambda_{b}$ eliminated with the use of (2.6), the load parameters $P$ and $N$ can be viewed as functions of the deformation parameters $\lambda_{a}$ and $\lambda_{z}$. It is shown in Fu et al. (2016) that the bifurcation condition for localized bulging is given by

$$
\Omega\left(\lambda_{a}, \lambda_{z}\right) \equiv \frac{\partial P}{\partial \lambda_{a}} \frac{\partial N}{\partial \lambda_{z}}-\frac{\partial P}{\partial \lambda_{z}} \frac{\partial N}{\partial \lambda_{a}}=0
$$

which states that the Jacobian determinant of the vector function $(P, N)$ vanishes. Alternatively, the variable $\lambda_{a}$ in the above expression can be replaced by the internal volume (Fu et al., 2018).

In the membrane approximation, it is convenient to define the wall thickness $H=B-A$, averaged radius $R_{m}=(A+B) / 2$, and a dimensionless thickness parameter $\alpha$ through

$$
\alpha=H / R_{m}
$$

The membrane limit is then characterized by $\alpha \rightarrow 0$, and it can be shown (Fu et al., 2016) that to leading order,

$$
P=\alpha \frac{w_{1}}{\lambda_{m} \lambda_{z}}, \quad N=2 \pi R_{m}^{2} \alpha\left(w_{2}-\frac{\lambda_{m} w_{1}}{2 \lambda_{z}}\right)
$$

where $\lambda_{m}$ is the azimuthal stretch at the mid-surface $R=R_{m}$ and $w_{1}=\partial w / \partial \lambda_{1}, w_{2}=$ $\partial w / \partial \lambda_{2}$. The neglected terms in both expressions are of order $\alpha^{3}$, which gives some inkling on why the membrane approximation is capable of giving accurate results even for tubes of moderate wall thicknesses. This was quantified in Fu et al. (2016).

On substituting (2.8) into (2.7), we obtain the following bifurcation condition in the membrane limit:

$$
\lambda_{m}\left(w_{1}-\lambda_{z} w_{12}\right)^{2}+\lambda_{z}^{2} w_{22}\left(w_{1}-\lambda_{m} w_{11}\right)=0
$$

where $w_{12}=\partial^{2} w / \partial \lambda_{1} \partial \lambda_{2}$ etc. The above condition was first recognized by Fu et al. (2008) as the condition for localized bulging, but the expression had already featured in the study of Haughton \& Ogden (1979a) as the condition for a sinusoidal mode with zero axial mode number to bifurcate from the uniformly inflated configuration. In the case of fixed $N$, this condition corresponds to the condition for the so-called limiting-point instability (Alexander, 1971). 


\subsection{Incremental equations}

To introduce the incremental equations, we denote by $B_{0}$ and $B_{e}$ the un-inflated configuration and the finitely deformed configuration determined in the previous section, respectively. The deformation gradient from $B_{0}$ and $B_{e}$ is denoted by $\bar{F}$ (which supersedes the notation $F$ in (2.1)) and the associated nominal stress by $\bar{S}$. A small amplitude axially symmetric perturbation is now applied to $B_{e}$, giving rise to the final configuration $B_{t}$, and the associated incremental displacement field $\delta \boldsymbol{x}$ is given by

$$
\delta \boldsymbol{x}=u(r, z) \boldsymbol{e}_{r}+v(r, z) \boldsymbol{e}_{z}
$$

The deformation gradient corresponding to the deformation $B_{0} \rightarrow B_{t}$ is denoted by $F$ and the associated nominal stress by $S$. We write $F=(I+\eta) \bar{F}$ so that $\eta$ denotes the deformation gradient associated with the incremental deformation $B_{e} \rightarrow B_{t}$. The divergence operator with respect to coordinates in $B_{0}$ and $B_{e}$ will be denoted by Div and div, respectively.

The incremental equilibrium equation can best be expressed in terms of the incremental stress tensor $\chi$ defined by

$$
\chi^{\mathrm{T}}=\bar{J}^{-1} \bar{F}(S-\bar{S}),
$$

where the superscript $\mathrm{T}$ stands for transpose, and $\bar{J}$ denotes the determinant of $\bar{F}$ (which is unity in the current case but is kept in the formula to maintain the generality of the formula). With the use of the identity $\operatorname{div} \bar{J}^{-1} \bar{F}=\mathbf{0}$ and the equilibrium equations $\operatorname{Div} \bar{S}=\mathbf{0}$ and Div $S=\mathbf{0}$, we obtain

$$
\operatorname{div} \chi^{\mathrm{T}}=\mathbf{0}
$$

For the current axi-symmetric deformation, only the equations corresponding to $i=2,3$ are not satisfied automatically, and they are given by

$$
\chi_{3 j, j}+\frac{1}{r}\left(\chi_{33}-\chi_{11}\right)=0, \quad \chi_{2 j, j}+\frac{1}{r} \chi_{23}=0 .
$$

For our weakly nonlinear analysis, we need expansions of $\chi_{i j}$ up to the quadratic order, and they are given by

$$
\chi_{i j}=B_{j i l k} \eta_{k l}+\bar{p} \xi_{j i}-p^{*}\left(\delta_{j i}-\xi_{j i}\right)+\frac{1}{2} B_{j i l k n m}^{2} \eta_{k l} \eta_{m n}+\cdots,
$$

where

$$
\xi_{j i}=\delta_{j i}-F_{A i}^{-1} \bar{F}_{j A}=\eta_{j i}-\eta_{j m} \eta_{m i}+\cdots .
$$

See, e.g., Fu \& Ogden (1999). In (2.14) the $\bar{p}$ and $p^{*}$ are the Lagrangian multipliers associated with the deformations $B_{0} \rightarrow B_{e}$ and $B_{e} \rightarrow B_{t}$, respectively. The $\eta_{k l}$ are the components of $\eta$ given by

$$
\eta=\left[\begin{array}{ccc}
\frac{u}{r} & 0 & 0 \\
0 & v_{z} & v_{r} \\
0 & u_{z} & u_{r}
\end{array}\right], \quad v_{z} \equiv \frac{\partial v}{\partial z}, \quad v_{r} \equiv \frac{\partial v}{\partial r} \text { etc }
$$


and $B_{j i l k}$ and $B_{\text {jilknm }}^{2}$ are the 1st- and 2nd-order instantaneous elastic moduli, the expressions of which can be found in Ogden (1984) or Fu \& Ogden (1999).

Due to the introduction of the extra variable $p^{*}$, the equilibrium equations are augmented by the incompressibility condition which can be expanded as

$$
\eta_{i i}-\frac{1}{2} \eta_{m n} \eta_{n m}+\ldots=0
$$

When (2.14) is substituted into (2.12), the resulting expression can be simplified by making use of the fact that $\operatorname{div} \xi=\mathbf{0}$, which follows from

$$
\operatorname{div} \xi=\operatorname{div}\left(I-\bar{F} F^{-1}\right)=-\operatorname{div}\left(\bar{J}^{-1} \bar{F} F^{-1}\right)=-\bar{J}^{-1} \operatorname{Div}\left(F^{-1}\right)=-\bar{J}^{-1} \operatorname{Div}\left(J F^{-1}\right)=\mathbf{0},
$$

where use has in turn been made of the relation $(2.15)$ and the identities $\operatorname{div}\left(\bar{J}^{-1} \bar{F}\right)=\mathbf{0}$ and $\operatorname{Div}\left(J F^{-1}\right)=\mathbf{0}$. More precisely, we shall replace $(2.12)$ by

$$
\boldsymbol{l} \equiv \operatorname{div} \chi^{\mathrm{T}}-\left(\bar{p}+p^{*}\right) \operatorname{div} \xi=\mathbf{0}
$$

in the subsequent derivations.

We also need to expand the boundary conditions to quadratic order. The external surface of the tube is assumed to be traction-free, and the inner surface is subjected to a hydrostatic pressure $P$. It then follows that on the inner surface we have

$$
S^{\mathrm{T}} F^{\mathrm{T}} \tilde{\boldsymbol{n}}=-P \tilde{\boldsymbol{n}}, \quad \bar{S}^{\mathrm{T}} \bar{F}^{\mathrm{T}} \boldsymbol{n}=-P \boldsymbol{n},
$$

where $\tilde{\boldsymbol{n}}$ is the unit outward normal to the inner surface in $B_{t}$ and $\boldsymbol{n}$ its counterpart in $B_{e}$. The two unit normals are related by Nanson's formula

$$
\bar{F}^{\mathrm{T}} \boldsymbol{n} d a=F^{\mathrm{T}} \tilde{\boldsymbol{n}} d \tilde{a},
$$

where $d a$ and $d \tilde{a}$ are area differentials in $B_{e}$ and $B_{t}$, respectively. With the use of these expressions, we obtain

$$
\begin{gathered}
\chi \boldsymbol{n} d a=S^{\mathrm{T}} \bar{F}^{\mathrm{T}} \boldsymbol{n} d a-\bar{S}^{\mathrm{T}} \bar{F}^{\mathrm{T}} \boldsymbol{n} d a=S^{\mathrm{T}} F^{\mathrm{T}} \tilde{\boldsymbol{n}} d \tilde{a}-\bar{S}^{\mathrm{T}} \bar{F}^{\mathrm{T}} \boldsymbol{n} d a=-P \tilde{\boldsymbol{n}} d \tilde{a}+P \boldsymbol{n} d a \\
=-P F^{-\mathrm{T}} \bar{F}^{\mathrm{T}} \boldsymbol{n} d a+P \boldsymbol{n} d a=P \xi^{\mathrm{T}} \boldsymbol{n} d a .
\end{gathered}
$$

Thus, we have

$$
\chi \boldsymbol{n}=-\sigma_{33} \xi^{\mathrm{T}} \boldsymbol{n}, \quad \text { on } r=a,
$$

where we have used the boundary condition $\left.\sigma_{33}\right|_{r=a}=-P$. The outer surface is traction free, and so the associated boundary condition is simply $\chi \boldsymbol{n}=0$ which can be replaced by $\chi \boldsymbol{n}=-\sigma_{33} \xi^{\mathrm{T}} \boldsymbol{n}$ since $\left.\sigma_{33}\right|_{r=b}=0$. Therefore, the boundary conditions on the inner and outer surfaces can both be written in the form

$$
\chi_{23}^{*}=0, \quad \chi_{33}^{*}=0, \quad \text { on } r=a, b,
$$

where $\chi^{*}$ is defined by

$$
\chi^{*}=\chi+\sigma_{33} \xi^{\mathrm{T}} .
$$

When the nonlinear terms are neglected, these boundary conditions are consistent with the boundary conditions (21) in Haughton \& Ogden (1979b). 


\section{Near-critical bulging solution}

We now look for an asymptotic solution for (2.17) and (2.18) subject to the boundary conditions (2.20). We use $\lambda_{a}$ as the control parameter in our post-bifurcation analysis. The $\lambda_{z}$ is either fixed or determined from the end condition $N=$ const. The idealized case of fixed $\lambda_{z}$ is used to approximate the case of fixed axial length which can be realized more easily experimentally or in Abaqus simulations. This approximation is expected to be valid in the initial stage of bulge growth although the two cases differ more and more as the bulge evolves further.

Guided by the analysis in Fu (2001), we may write

$$
\lambda_{a}=\lambda_{a c r}+\varepsilon \lambda_{0},
$$

and define a far distance variable $s$ through

$$
s=\sqrt{\varepsilon} z,
$$

where $\lambda_{0}$ is a constant and $\varepsilon$ is a small positive parameter characterizing the order of deviation of $\lambda_{a}$ from its critical value $\lambda_{a c r}$. Note that as a result of (3.1), the moduli $B_{j i l k}$ and $B_{j i l k n m}^{2}$, the deformed radii $a$ and $b$, and $\lambda_{z}$ (if not fixed) must all be expanded in terms of $\varepsilon$ as well, but these expansions are not written out for the sake of brevity. Again guided by the scalings given by $\mathrm{Fu}$ (2001), we may assume

$$
u=O(\sqrt{\varepsilon} v), \quad p^{*}=O(\sqrt{\varepsilon} v),
$$

and that $v$ is of order $\sqrt{\varepsilon}$. Thus, we look for an asymptotic solution of the form

$$
\begin{aligned}
& u=\varepsilon\left\{u^{(1)}(r, s)+\varepsilon u^{(2)}(r, s)+\varepsilon^{2} u^{(3)}(r, s)+\cdots\right\}, \\
& v=\sqrt{\varepsilon}\left\{v^{(1)}(r, s)+\varepsilon v^{(2)}(r, s)+\varepsilon^{2} v^{(3)}(r, s)+\cdots\right\}, \\
& p^{*}=\varepsilon\left\{p^{(1)}(r, s)+\varepsilon p^{(2)}(r, s)+\varepsilon^{2} p^{(3)}(r, s)+\cdots\right\},
\end{aligned}
$$

where all the functions on the right hands are to be determined from successive approximations.

In the linear analysis, the incremental pressure $p^{*}$ can be eliminated by subtracting the cross-differentiations of the two equilibrium equations in (2.18), that is from $l_{3,2}-l_{2,3}=0$. In the current nonlinear setting, it is no longer possible to eliminate $p^{*}$ completely, but the above manipulation still helps since it can at least eliminate $p^{*}$ from the first and second orders.

On substituting (3.3) into $l_{3,2}-l_{2,3}=0$, the incompressibility condition (2.17), the boundary conditions (2.20), and then equating the coefficients of like powers of $\varepsilon$, we obtain a hierarchy of boundary value problems. To leading order, we obtain

$$
\frac{d}{d r} \frac{1}{r} \frac{d}{d r} r \zeta(r) v_{r s}^{(1)}=0, \quad v_{s}^{(1)}+\frac{1}{r} \frac{d}{d r} r u^{(1)}=0, \quad a<r<b,
$$


and

$$
v_{r s}^{(1)}=0, \quad \frac{1}{r} \frac{d}{d r} r \zeta(r) v_{r s}^{(1)}=0, \quad \text { on } r=a, b,
$$

where $\zeta(r)=B_{3232}$ and $v_{r s}^{(1)}=\partial^{2} v^{(1)} / \partial r \partial s$. Note that $a$ and $b$ are now the inner and outer radii at the critical configuration.

Using $(3.4)_{2}$ to eliminate $v_{s}^{(1)}$ from $(3.4)_{1}$ and (3.5), we obtain

$$
\begin{gathered}
\mathcal{L}\left[u^{(1)}\right]=0, \quad a<r<b, \\
\mathcal{B}_{1}\left[u^{(1)}\right]=0, \quad \mathcal{B}_{2}\left[u^{(1)}\right]=0, \quad \text { on } r=a, b,
\end{gathered}
$$

where the three differential operators are defined by

$$
\mathcal{L}[u]=\frac{d}{d r} \frac{1}{r} \frac{d}{d r} r \zeta(r) \frac{d}{d r} \frac{1}{r} \frac{d}{d r} r u, \quad \mathcal{B}_{1}[u]=\frac{1}{r} \frac{d}{d r} r \zeta(r) \frac{d}{d r} \frac{1}{r} \frac{d}{d r} r u, \quad \mathcal{B}_{2}[u]=\frac{d}{d r} \frac{1}{r} \frac{d}{d r} r u .
$$

On integrating (3.4), we find that $v^{(1)}$ and $u^{(1)}$ must be a linear combination of the independent solutions

$$
1, \quad \hat{\kappa}_{1}(r), \quad \hat{\kappa}_{2}(r)
$$

and

$$
r, \frac{1}{r}, \quad \kappa_{1}(r), \quad \kappa_{2}(r)
$$

respectively, where

$$
\begin{array}{ccl}
\hat{\kappa}_{1}(r)=\int_{a}^{r} \frac{s}{\zeta(s)} d s, & \hat{\kappa}_{2}(r)=\int_{a}^{r} \frac{1}{s \zeta(s)} d s, \\
\kappa_{1}(r)=\frac{1}{r} \int_{a}^{r} t \hat{\kappa}_{1}(t) d t & \kappa_{2}(r)=\frac{1}{r} \int_{a}^{r} t \hat{\kappa}_{2}(t) d t .
\end{array}
$$

However, on substituting this general solution into the boundary conditions (3.5), we find that the coefficients of $\hat{\kappa}_{1}, \hat{\kappa}_{2}, \kappa_{1}, \kappa_{2}$ must necessarily vanish, and so $u^{(1)}$ and $v^{(1)}$ take the reduced form

$$
v^{(1)}=-A_{1}(s), \quad u^{(1)}=c_{1}(s) r+c_{2}(s) \frac{1}{r},
$$

where $c_{1}(s)$ and $c_{2}(s)$ are arbitrary functions, and $A_{1}^{\prime}(s)=2 c_{1}(s)$.

At second order, we find that $u^{(2)}$ and $v^{(2)}$ satisfy the governing equations

$$
\frac{d}{d r} \frac{1}{r} \frac{d}{d r} r \zeta(r) v_{r s}^{(2)}=h_{1}, \quad v_{s}^{(2)}+\frac{1}{r} \frac{d}{d r} r u^{(2)}=h_{2}, \quad a<r<b
$$

and boundary conditions

$$
v_{r s}^{(2)}=h_{3}, \quad \frac{1}{r} \frac{d}{d r} r \zeta(r) v_{r s}^{(2)}=h_{4}, \quad \text { on } r=a, b,
$$

where the inhomogeneous terms $h_{1}, h_{2}, h_{3}$ and $h_{4}$ on the right hand sides involve the leading order solution $u^{(1)}$. It turns out that $h_{1}$ is linear in terms of $c_{1}^{\prime}(s)$ and $c_{2}^{\prime}(s)$, taking the form

$$
h_{1}=-c_{1}^{\prime}(s) \omega_{1}(r)-c_{2}^{\prime}(s) \omega_{2}(r)
$$


where $\omega_{1}(r)$ and $\omega_{2}(r)$ are as defined in Fu et al. (2016). Solving (3.9) $)_{1}$ then yields

$$
v^{(2)}=-A_{2}(s)-A_{3}(s) \hat{\kappa}_{1}(r)-A_{4}(s) \hat{\kappa}_{2}(r)-c_{1}^{\prime}(s) \hat{\kappa}_{3}(r)-c_{2}^{\prime}(s) \hat{\kappa}_{4}(r)
$$

where

$$
\hat{\kappa}_{3}(r)=\int_{a}^{r} \frac{1}{x \zeta(x)} \int_{a}^{x} t \int_{a}^{t} \omega_{1}(s) d s d t d x, \quad \hat{\kappa}_{4}(r)=\int_{a}^{r} \frac{1}{x \zeta(x)} \int_{a}^{x} t \int_{a}^{t} \omega_{2}(s) d s d t d x .
$$

It is seen that the first three terms in (3.12) are the complementary solution whereas the last two terms represent a particular integral. In view of the fact that the boundary conditions (3.10) does not involve $u^{(2)}$, we may now substitute (3.8) and (3.12) into (3.10) and obtain a matrix equation of the form $M \boldsymbol{d}=\mathbf{0}$ where $M$ is a $4 \times 4$ matrix which is not written out here for the sake of brevity, and $\boldsymbol{d}$ is the column vector formed from the four unknown functions $c_{1}^{\prime \prime}(s), c_{2}^{\prime \prime}(s), A_{3}^{\prime}(s), A_{4}^{\prime}(s)$. It then follows that $\operatorname{det} M=0$ must be satisfied for a non-trivial solution to exist. This condition is simply the bifurcation condition, and is verified numerically to be equivalent to (2.7) as pointed out by Fu et al. (2016). Also, $c_{2}^{\prime \prime}(s), A_{3}^{\prime}(s), A_{4}^{\prime}(s)$ can be expressed as constant multiples of $c_{1}^{\prime \prime}(s)$. In particular, after integrating the expression for $c_{2}^{\prime \prime}(s)$ twice, we obtain

$$
c_{2}(s)=\Gamma c_{1}(s)
$$

for localized solutions, where $\Gamma$ is a constant depending on tube wall thickness and the material model used.

Once $u^{(1)}, v^{(1)}$ and $v^{(2)}$ are known, the leading order incremental pressure $p^{(1)}$ can be obtained by equating the coefficients of $\varepsilon^{3 / 2}$ in $(2.13)_{2}$ and then integrating the resulting equation with respect to $s$. We thus obtain

$$
\begin{gathered}
p^{(1)}=\left\{B_{2211}-2 B_{2222}+B_{2233}+2 B_{3223}+r B_{3223}^{\prime}-\int_{0}^{r} \omega_{1}(t) d t+r \bar{p}^{\prime}\right\} c_{1}(s) \\
+\frac{1}{r^{2}}\left\{B_{2211}-2 B_{2233}+r B_{3223}^{\prime}-r^{2} \int_{0}^{r} \omega_{2}(t) d t+r \bar{p}^{\prime}\right\} c_{2}(s)-2 \int_{-\infty}^{s} A_{3}(s) d s .
\end{gathered}
$$

Finally, $u^{(2)}$ can be determined from the incompressibility condition (3.9) as

$$
\begin{gathered}
u^{(2)}=c_{3}(s) r+\frac{1}{r} c_{4}(s)+A_{3}^{\prime}(s) \kappa_{1}(r)+A_{4}^{\prime}(s) \kappa_{2}(r) \\
+c_{1}^{\prime \prime}(s) \kappa_{3}(r)+c_{4}^{\prime \prime}(s) \kappa_{4}(r)+\frac{3}{2} r c_{1}^{2}(s)-\frac{1}{2 r^{3}} c_{2}^{2}(s),
\end{gathered}
$$

where $c_{4}(s)$ is a new arbitrary function arising from integration, $c_{3}(s)=A_{2}^{\prime}(s) / 2$, and

$$
\kappa_{3}(r)=\frac{1}{r} \int_{a}^{r} t \hat{\kappa}_{3}(t) d t, \quad \kappa_{4}(r)=\frac{1}{r} \int_{a}^{r} t \hat{\kappa}_{4}(t) d t .
$$

This then completes the first and second order solutions, and we are now in a position to derive the amplitude equation. 
At third order, $u^{(3)}$ and $v^{(3)}$ satisfy a boundary value problem similar to (3.9)-(3.10). However, the inhomogeneous term in the counterpart of $(3.9)_{1}$ now contains nonlinear terms, and as a result the solution takes the form

$$
v^{(3)}=-A_{7}(s)-A_{5}(s) \hat{\kappa}_{1}(r)-A_{6}(s) \hat{\kappa}_{2}(r)-c_{3}^{\prime}(s) \hat{\kappa}_{3}(r)-c_{4}^{\prime}(s) \hat{\kappa}_{4}(r)+\kappa_{5}(r, s),
$$

where the first five terms are similar to those in (3.12) with $A_{5}(s), A_{6}(s), A_{7}(s)$ to be determined at higher order, and $\kappa_{5}(r, s)$ is a particular solution associated with the nonlinear terms mentioned above. Substituting (3.18) and the lower order solutions into the associated boundary conditions, we obtain a matrix equation of the form $M \boldsymbol{e}=\boldsymbol{f}$, where the column vector $\boldsymbol{e}$ is formed from the four unknown functions $c_{3}^{\prime \prime}(s), c_{4}^{\prime \prime}(s), A_{5}^{\prime}(s), A_{6}^{\prime}(s)$, and the right hand side $\boldsymbol{f}$ contains lower order solutions and can be expressed entirely in terms of $c_{1}(s)$. Since $M$ is singular, a solvability condition, given by $\boldsymbol{f} \cdot \boldsymbol{h}=0$, must be satisfied by $\boldsymbol{f}$, where $\boldsymbol{h}$ is a solution of $M^{T} \boldsymbol{h}=\mathbf{0}$. After some rearrangement, we find that the solvability condition takes the form

$$
c_{1}^{\prime \prime \prime \prime}(s)+\lambda_{0} k_{1} c_{1}^{\prime \prime}(s)+k_{2}\left(c_{1}^{2}(s)\right)^{\prime \prime}=0,
$$

where $k_{1}$ and $k_{2}$ are constants whose expressions are obtained with the aid of Mathematica (Wolfram Research Inc, 2019), but are not presented here for the sake of brevity.

The above method of deriving the amplitude equation is conceptually simple, but the expressions obtained for $k_{1}$ and $k_{2}$ are quite lengthy. As is usual in this kind of problems, there is no need to actually solve the differential equation for $v^{(3)}$ if the solvability condition is all we need to find. One alternative method is to make use of the fact that for arbitrary functions $f(r)$ and $g(r)$ that are sufficiently smooth, there exists the identity

$$
\int_{a}^{b} r g \mathcal{L}[f]-r f \mathcal{L}[g] d r=\left.\left(r g \mathcal{B}_{1}[f]-r f \mathcal{B}_{1}[g]+(r f)^{\prime} \zeta(r) \mathcal{B}_{2}[g]-(r g)^{\prime} \zeta(r) \mathcal{B}_{2}[f]\right)\right|_{a} ^{b},
$$

which implies that the operator $\mathcal{L}$ is self-adjoint. In particular, taking $g$ to be any solution of (3.6) subject to $(3.7)$, and $f=u^{(m)}, m=2,3$, we obtain

$$
\int_{a}^{b} r g \mathcal{L}\left[u^{(m)}\right] d r=\left.\left(r g \mathcal{B}_{1}\left[u^{(m)}\right]-(r g)^{\prime} \zeta(r) \mathcal{B}_{2}\left[u^{(m)}\right]\right)\right|_{a} ^{b}
$$

Note that $\mathcal{L}\left[u^{(m)}\right], \mathcal{B}_{1}\left[u^{(m)}\right]$ and $\mathcal{B}_{2}\left[u^{(m)}\right]$ are each equal to an expression that only involves lower order solutions. Thus, taking $m=2$, and $g=r$ or $1 / r$ in turn in (3.21), we would obtain a matrix equation of the form $\hat{M} \hat{\boldsymbol{d}}=\mathbf{0}$ where $\hat{M}$ is a $2 \times 2$ matrix, and $\hat{\boldsymbol{d}}$ is the column vector formed from the two functions $c_{1}^{\prime \prime}(s)$ and $c_{2}^{\prime \prime}(s)$. The bifurcation condition is then also given by $\operatorname{det} \hat{M}=0$, which provides a check on the bifurcation condition $\operatorname{det} M=0$ obtained earlier.

On the other hand, by taking $m=3$, and $g=r$ and $1 / r$ in turn in (3.21), we obtain a matrix equation of the form $\hat{M} \hat{\boldsymbol{e}}=\hat{\boldsymbol{f}}$, where the 2-vector $\hat{\boldsymbol{e}}$ is formed from the two functions $c_{3}^{\prime \prime}(s)$ and $c_{4}^{\prime \prime}(s)$, and the right hand side $\hat{\boldsymbol{f}}$ only involves leading and second order solutions. 
Since $\hat{M}$ is singular, the vector function $\hat{\boldsymbol{f}}$ must satisfy the solvability condition $\hat{\boldsymbol{f}} \cdot \hat{\boldsymbol{h}}=0$ with $\hat{\boldsymbol{h}}$ denoting a solution of $\hat{M}^{T} \hat{\boldsymbol{h}}=\mathbf{0}$. This should and does indeed give the same amplitude equation (3.19), but with simpler expressions for the coefficients.

Focusing on localized solutions that satisfy the decaying conditions $c_{1}(s) \rightarrow 0$ as $s \rightarrow \pm \infty$, we may integrate (3.19) twice to obtain

$$
c_{1}^{\prime \prime}(s)=\lambda_{0} k_{1} c_{1}(s)+k_{2} c_{1}^{2}(s) .
$$

In the bulged configuration, the radius at the mid-surface $r=r_{m}$, denoted by $\tilde{r}(z)$, is given by

$$
\tilde{r}(z)=r_{m}+u\left(r_{m}, z\right)=r_{\mathrm{cr}}+\varepsilon r_{1}+\varepsilon\left(r_{\mathrm{cr}}+\frac{\Gamma}{r_{\mathrm{cr}}}\right) c_{1}(s),
$$

where $\Gamma$ is defined by (3.14) and the near-critical expansion $r_{m}=r_{\mathrm{cr}}+\varepsilon r_{1}$ is the counterpart of (3.1) for $r_{m}$ (the connection between $r_{1}$ and $\lambda_{0}$, for instance, will be derived shortly in the membrane limit). Also, here and hereafter we consistently neglect terms of order $\varepsilon^{2}$. It is then appropriate to define a normalized amplitude $A(s)$ through

$$
A(s)=\left(r_{\mathrm{cr}}+\frac{\Gamma}{r_{\mathrm{cr}}}\right) c_{1}(s)
$$

In terms of $A$ the amplitude equation (3.22) takes the form

$$
A^{\prime \prime}(s)=k_{3} r_{1} A(s)+k_{4} A^{2}(s)
$$

where

$$
k_{3}=\left(\lambda_{0} / r_{1}\right) k_{1}, \quad k_{4}=k_{2}\left(r_{\mathrm{cr}}+\frac{\Gamma}{r_{\mathrm{cr}}}\right)^{-1} .
$$

This amplitude equation is of the same form as that obtained under the membrane assumption, as will be discussed further later. It takes the same form as the ordinary differential equation governing the shape of steady solitary wave solutions of the well-known Kortewedde Vries $(\mathrm{KdV})$ equation $u_{t}-6 u u_{x}+u_{x x x}=0$. The localized bulging solution that we are looking for is simply a standing solitary wave, and is given by

$$
A(s)=-\frac{3 r_{1} k_{3}}{2 k_{4}} \operatorname{sech}^{2}\left(\frac{1}{2} \sqrt{r_{1} k_{3}} s\right) .
$$

We note that the above solution is only defined if $r_{1} k_{3}$ is positive. Since a positive value of $r_{1}$ corresponds to a higher value of $r_{m}$ above its critical value (and hence a higher pressure), it follows that bifurcation into the above localized solution is supercritical if $k_{3}>0$ and subcritical if $k_{3}<0$. Assume that $r_{1} k_{3}>0$. The bifurcation solution (3.26) then represents a bulge (a bright soliton) if $k_{4}<0$ or a neck (a dark soliton) if $k_{4}>0$. All experiments that have been carried out so far for isotropic rubber tubes seem to indicate that the first bifurcation value of $\lambda_{a}$ corresponds to localized bulging and the bifurcation is subcritical. This will partially be confirmed in the next section by numerical results based on our analysis 
although it will be shown that localized necking is also possible in some extreme cases. If $k_{3}$ happened to be zero, then higher order terms would need to be included by re-scaling the independent and dependent variables.

As a first check on the validity of the amplitude equation (3.22), we note that it admits a constant solution given by

$$
c_{1}(s)=-\frac{k_{1}}{k_{2}} \lambda_{0}
$$

Corresponding to this solution the azimuthal stretch on the inner surface is given by

$$
\tilde{\lambda}_{a}=\lambda_{a}+\frac{u(a, z)}{A}=\lambda_{a \mathrm{cr}}+\varepsilon \lambda_{0}-\varepsilon\left(\lambda_{a \mathrm{cr}}+\frac{\Gamma}{A^{2} \lambda_{a \mathrm{cr}}}\right) \frac{k_{1}}{k_{2}} \lambda_{0}+\cdots .
$$

In the case of fixed $N$, the bifurcation stretch $\lambda_{a c r}$ corresponds to the pressure maximum, and the azimuthal stretch $\tilde{\lambda}_{a}$ given by the above expression and by (3.1) are two possible constant solutions corresponding to the same pressure. With the aid of the local expansion

$$
P=P_{\mathrm{cr}}+\left.\frac{1}{2} \frac{d^{2} P}{d \lambda_{a}^{2}}\right|_{\lambda_{a}=\lambda_{a \mathrm{cr}}}\left(\lambda_{a}-\lambda_{a \mathrm{cr}}\right)^{2}+\cdots,
$$

it then follows that

$$
\left(\lambda_{a}-\lambda_{a \mathrm{cr}}\right)^{2}=\left(\tilde{\lambda}_{a}-\lambda_{a \mathrm{cr}}\right)^{2}, \quad \Longrightarrow \quad \lambda_{a}-\lambda_{a \mathrm{cr}}=-\left(\tilde{\lambda}_{a}-\lambda_{a \mathrm{cr}}\right)
$$

and hence that

$$
\left(\lambda_{a \mathrm{cr}}+\frac{\Gamma}{A^{2} \lambda_{a \mathrm{cr}}}\right) \frac{k_{1}}{k_{2}}=2 .
$$

This identity is monitored in all our numerical computations for fixed $N$.

\section{Numerical results}

In this section, we present some illustrative numerical results and compare them with the corresponding membrane theory results and Abaqus simulations (ABAQUS, 2013). Our expressions for the coefficients in the amplitude equation are analytical, but contain a number of definite integrals that cannot be evaluated analytically. These integrals are evaluated numerically using the following strategy. Take as an example the evaluation of $\kappa_{3}(r)$ defined by $(3.17)_{1}$ together with $(3.13)_{1}$. We first discretize the interval $[a, b]$ with equally spaced node points $a<r_{1}<r_{2}<\cdots r_{n}=b$. The integrals $\int_{a}^{r_{i}} \omega_{1}(s) d s(i=1,2, \ldots, n)$ are evaluated numerically using the built-in command NIntegrate on Mathematica. The function $\int_{a}^{t} \omega_{1}(s) d s(a \leq t \leq b)$ is then defined by the built-in command Interpolation on Mathematica. Further integrals involving this numerically defined function are determined in a similar manner. To avoid errors arising from differentiation of such numerically defined functions, derivatives of such functions are eliminated using analytical expressions wherever possible. For instance, $\kappa_{3}^{\prime}(r)$ is eliminated with the use of $\kappa_{3}^{\prime}(r)=-\kappa_{3}(r) / r+\hat{\kappa}_{3}(r)$ that 
follows from $(3.17)_{1}$, and $\kappa_{3}^{\prime \prime}(r)$ is eliminated by differentiating this analytical expression again. The number of discretizing points is varied to test accuracy. It is found that choosing this number to be 200 gives numerical results with at least three significant figures and the identity (3.28) is satisfied with a relative error less than $1 \%$ except when the two bifurcation points get very close.

We shall assume that the strain energy function is given by the Gent material model:

$$
W=-\frac{\mu}{2} J_{m} \ln \left(1-\frac{\lambda_{1}^{2}+\lambda_{2}^{2}+\lambda_{3}^{2}-3}{J_{m}}\right),
$$

where $\mu$ is the ground state shear modulus and $J_{m}$ is a material constant characterizing material extensibility which we take to be 97.2, a typical value for rubber materials (Gent, 1996). To facilitate comparison, we shall scale all length variables/parameters by the averaged radius $R_{m}$, the internal pressure by $\alpha \mu$, and the resultant axial force by $2 \pi R_{m}^{2} \alpha$; see (2.8). In Figure 1(a), we have shown a representative solution of the bifurcation condition (2.7) when $\alpha=0.01$ together with two possible loading paths $N\left(\lambda_{a}, \lambda_{z}\right)=0$ and $N\left(\lambda_{a}, \lambda_{z}\right)=3.25$, respectively. Figure 1(b) shows the variation of $P$ with respect to $\lambda_{a}$ associated with the condition $N\left(\lambda_{a}, \lambda_{z}\right)=0$. We note that the pressure maximum and minimum correspond to the intersections A and B in Figure 1(a), respectively. The two bifurcation points A and $\mathrm{B}$ are associated with localized bulging and necking, respectively, and they get closer and closer as $N$ is increased, coalescing when $N$ is approximately equal to 3.25. Thus, for the case of fixed $N$, localized bulging is only possible for $N$ less than 3.25. On the other hand, if it is the axial stretch that is fixed during inflation, then localized bulging is only possible for $\lambda_{z}$ less than 3.61, the value at which the two bifurcation points coalesce. We do not make precise statements about the lower bounds for $N$ or $\lambda_{z}$. As $N$ or $\lambda_{z}$ is reduced, a point will be reached where localized bulging gives way to periodic buckling. Determination of this boundary is beyond the scope of this paper.

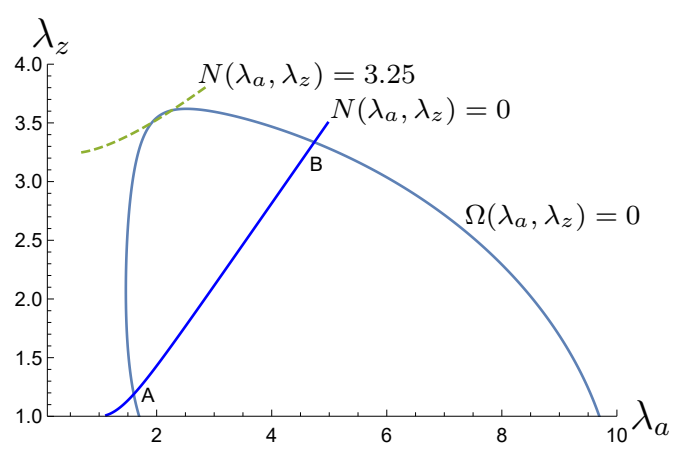

(a)

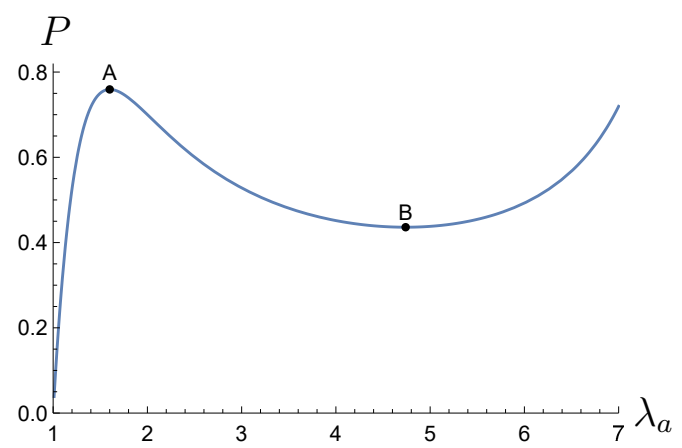

(b)

Figure 1: (a) Solution of the bifurcation condition (2.7) and two possible loading paths with fixed $N$; (b) Relation between $P$ and $\lambda_{a}$ in uniform inflation with $N=0$. The curve $N=0$ in (a) can be viewed as a loading path and the points $\mathrm{A}$ and $\mathrm{B}$ on this path correspond to points $\mathrm{A}$ and $\mathrm{B}$ in $(\mathrm{b})$.

In our Abaqus simulations, all tubes have averaged radius equal to 10 units and total 
length equal to 300 units unless otherwise stated. We have also considered the total length equal to 500, but the numerical results obtained are found to be graphically indistinguishable from when the length is 300 units. To ensure that localized bulging initiates from the middle point of the tube, a small section with length 20 units around the middle point is weakened by taking its shear modulus to be 0.9995 times the modulus for the rest of the tube. For the case of fixed $N$, the ends are allowed to move freely in the axial direction but are prevented from moving in the radial direction. For the case of fixed total length, neither end is allowed to move in the axial direction after applying the pre-stretch, but is free to move in the radial direction. The $r_{\infty}$ is taken to be the averaged radius near the ends. This should provide a very good approximation except when the rapid propagation stage is reached. The critical stretch $\lambda_{a c r}$ given by Abaqus simulations typically differs from its exact value by around $1 \%$.

Under the membrane assumption, the principal stretches at the center of the bulge (i.e. $z=0$ ) can be determined by solving two algebraic equations whether it is the $N$ or $\lambda_{z}$ that is fixed ( $\mathrm{Fu}$ et al., 2008). In the case of fixed length, these principal stretches can be determined by using the iteration procedure explained in Wang et al. (2019). Thus, in all three cases the amplitude diagrams can be obtained semi-analytically.

\subsection{Validation of the coefficients $k_{3}$ and $k_{4}$ by comparing with the membrane theory}

For the primary deformation, it can be shown that

$$
\lambda_{a}=\left(r_{m}-\frac{\alpha}{2 r_{m} \lambda_{z}}\right)\left(1-\frac{1}{2} \alpha\right)^{-1}
$$

where $r_{m}$ is the constant radius of the mid-surface in the finitely inflated, non-bulged configuration when $\lambda_{a}$ is given by (3.1), and is the main parameter used in the membrane theory. When describing localized bulging solutions, we rewrite $r_{m}$ as $r_{\infty}$ to signify the fact that it is the (scaled) uniform radius at infinity (but for a sufficiently long tube it is the radius near the ends). If $r_{\infty}=r_{\text {cr }}+\varepsilon r_{1}$ as in Fu et al. (2008), then

$$
\lambda_{a}=\left(r_{\mathrm{cr}}-\frac{\alpha}{2 r_{\mathrm{cr}} \lambda_{z}}\right)\left(1-\frac{1}{2} \alpha\right)^{-1}+\varepsilon r_{1}\left(1-\frac{1}{2} \alpha\right)^{-1},
$$

where we have neglected terms of order $\alpha \varepsilon$. Comparing this expression with (3.1) gives the connections

$$
\lambda_{a \mathrm{cr}}=\left(r_{\mathrm{cr}}-\frac{\alpha}{2 r_{\mathrm{cr}} \lambda_{z}}\right)\left(1-\frac{1}{2} \alpha\right)^{-1}, \quad \lambda_{0}=r_{1}\left(1-\frac{1}{2} \alpha\right)^{-1} .
$$

If we use the critical value $r_{\mathrm{cr}}$ from the membrane theory to compute the right hand side of $(4.4)_{1}$, the result will differ from $\lambda_{a c r}$ determined from the exact bifurcation condition (2.7). The accuracy of the membrane theory is then determined by how close these two values are. Such a quantitative assessment has previously been carried out in Fu et al. (2016) and will not be repeated here. It is the second connection in (4.4) that will be used in the comparison of the amplitude equations. 
Table 1: Linear and non-linear coefficients given by the exact theory and their membrane approximations: fixed $\lambda_{z}=1.5$ and Gent model.

\begin{tabular}{ccccccc}
\hline & $k_{3}$ & $k_{3 \mathrm{mem}}$ & $\left|1-k_{3} / k_{3 \mathrm{mem}}\right|$ & $k_{4}$ & $k_{4 \mathrm{mem}}$ & $1-k_{4} / k_{4 \mathrm{mem}} \mid$ \\
\hline$\alpha=0.5$ & -0.9888 & -1.060 & $6.7 \%$ & -0.5928 & -0.5854 & $1.3 \%$ \\
\hline$\alpha=0.4$ & -1.001 & -1.060 & $5.6 \%$ & -0.5904 & -0.5854 & $0.85 \%$ \\
\hline$\alpha=0.3$ & -1.015 & -1.060 & $4.2 \%$ & -0.5891 & -0.5854 & $0.63 \%$ \\
\hline$\alpha=0.2$ & -1.0291 & -1.060 & $2.9 \%$ & -0.5884 & -0.5854 & $0.51 \%$ \\
\hline$\alpha=0.1$ & -1.044 & -1.060 & $1.5 \%$ & -0.5880 & -0.5854 & $0.44 \%$ \\
\hline$\alpha=0.05$ & -1.052 & -1.060 & $0.75 \%$ & -0.5879 & -0.5854 & $0.43 \%$ \\
\hline$\alpha=0.01$ & -1.058 & -1.060 & $0.19 \%$ & -0.5879 & -0.5854 & $0.43 \%$ \\
\hline
\end{tabular}

On the other hand, according to the weakly nonlinear analysis under the membrane assumption, Fu et al. (2008), the radius at the mid-surface in the bulged configuration is given by

$$
r=r_{\mathrm{cr}}+\varepsilon r_{1}+\varepsilon y
$$

where in terms of the notations used in the current paper $y$ satisfies the differential equation

$$
y^{\prime \prime}(s)=\frac{1}{\lambda_{z}^{2}} \omega^{\prime}\left(r_{\mathrm{cr}}\right) r_{1} y+\frac{3}{2 \lambda_{z}^{2}} \gamma\left(r_{\mathrm{cr}}\right) y^{2}
$$

and the coefficient functions $\omega\left(r_{\infty}\right)$ and $\gamma\left(r_{\infty}\right)$ are as defined in Fu et al. (2008). Comparing (4.6) with (3.24) then shows that we should have

$$
k_{3}=\frac{1}{\lambda_{z}^{2}} \omega^{\prime}\left(r_{\mathrm{cr}}\right)+O(\alpha), \quad k_{4}=\frac{3}{2 \lambda_{z}^{2}} \gamma\left(r_{\mathrm{cr}}\right)+O(\alpha)
$$

Although it is not possible to establish this connection analytically due to the complex nature of the expressions for $k_{3}$ and $k_{4}$, we can verify this numerically. We shall denote the first terms on the right hand sides of (4.7) by $k_{3 \mathrm{mem}}$ and $k_{4 \mathrm{mem}}$, respectively, and refer to them as membrane approximations. In Table 1, we have shown values of these coefficients for the case when $\lambda_{z}$ is fixed to be 1.5. The corresponding results for the case of fixed $N=0$ are given in Table 2. It is seen that in the limit $\alpha \rightarrow 0$, our amplitude equation (3.25) does indeed tend to its counterpart in the membrane theory. 
Table 2: Linear and non-linear coefficients given by the exact theory and their membrane approximations: fixed $N=0$ and Gent model.

\begin{tabular}{ccccccc}
\hline & $k_{3}$ & $k_{3 \mathrm{mem}}$ & $\left|1-k_{3} / k_{3 \mathrm{mem}}\right|$ & $k_{4}$ & $k_{4 \mathrm{mem}}$ & $\left|1-k_{4} / k_{4 \mathrm{mem}}\right|$ \\
\hline$\alpha=0.5$ & -2.718 & -3.217 & $16 \%$ & -1.574 & -1.609 & $2.2 \%$ \\
\hline$\alpha=0.4$ & -2.817 & -3.217 & $12 \%$ & -1.584 & -1.609 & $1.6 \%$ \\
\hline$\alpha=0.3$ & -2.920 & -3.217 & $9.2 \%$ & -1.595 & -1.609 & $0.87 \%$ \\
\hline$\alpha=0.2$ & -3.023 & -3.217 & $6.0 \%$ & -1.604 & -1.609 & $0.31 \%$ \\
\hline$\alpha=0.1$ & -3.123 & -3.217 & $2.9 \%$ & -1.610 & -1.609 & $0.062 \%$ \\
\hline$\alpha=0.05$ & -3.171 & -3.217 & $1.4 \%$ & -1.612 & -1.609 & $0.19 \%$ \\
\hline$\alpha=0.01$ & -3.208 & -3.217 & $0.28 \%$ & -1.612 & -1.609 & $0.19 \%$ \\
\hline
\end{tabular}

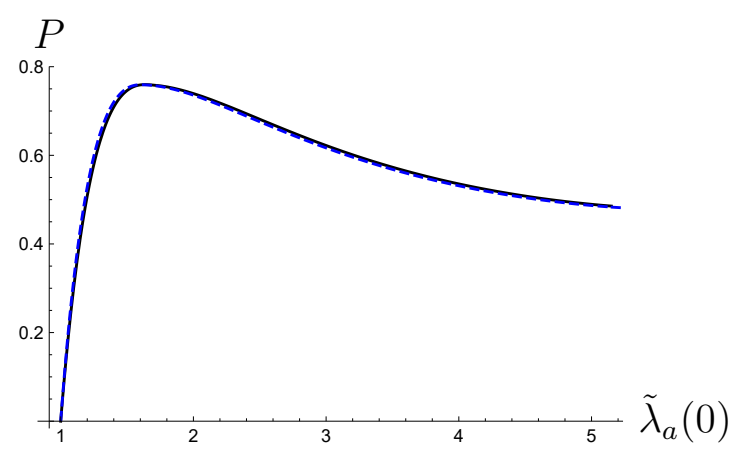

(a)

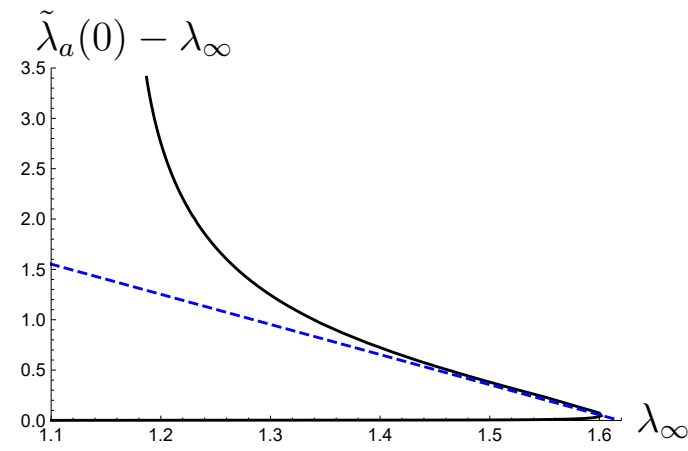

(b)

Figure 2: Amplitude diagrams for a thin-walled tube with $\alpha=0.04$ and $N=0$. The solid lines in both (a) and (b) are numerical simulation results using Abaqus, the dashed line in (a) is the result based on the membrane assumption, and the dashed line (b) is the weakly nonlinear result given by the full 3D theory.

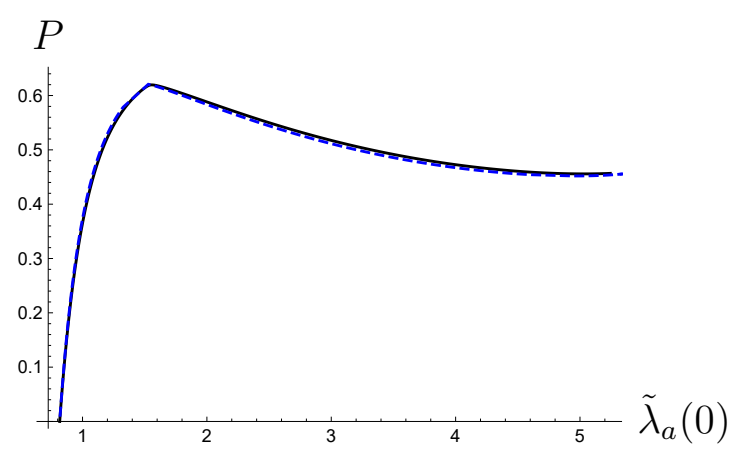

(a)

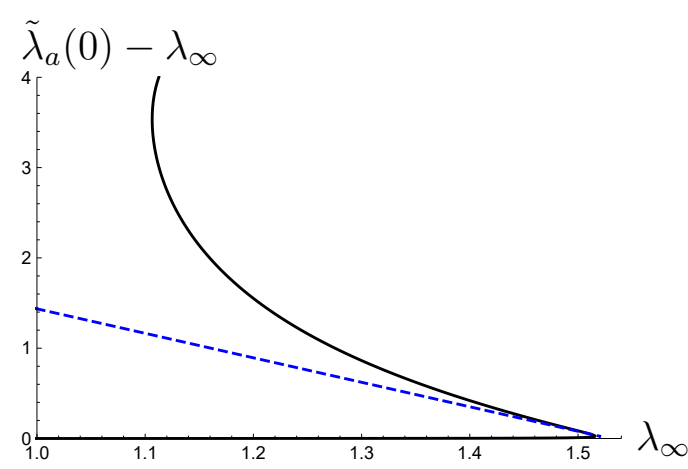

(b)

Figure 3: Amplitude diagrams for a thin-walled tube with $\alpha=0.04$ and $\lambda_{z}$ fixed to be 1.5. The solid lines in both (a) and (b) are numerical simulation results using Abaqus, the dashed line in (a) is the result based on the membrane assumption, and the dashed line (b) is the weakly nonlinear result given by the full 3D theory. 


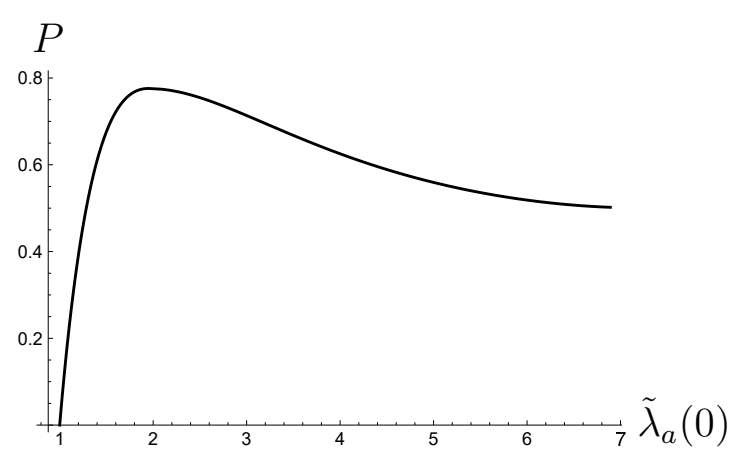

(a)

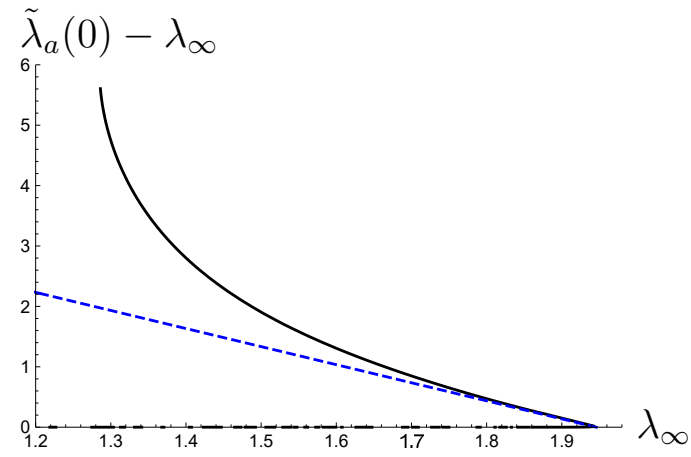

(b)

Figure 4: Amplitude diagrams for a thick-walled tube with $\alpha=0.5$ and $N=0$. The solid lines in both (a) and (b) are numerical simulation results using Abaqus and the dashed line (b) is the weakly nonlinear result given by the full 3D theory.

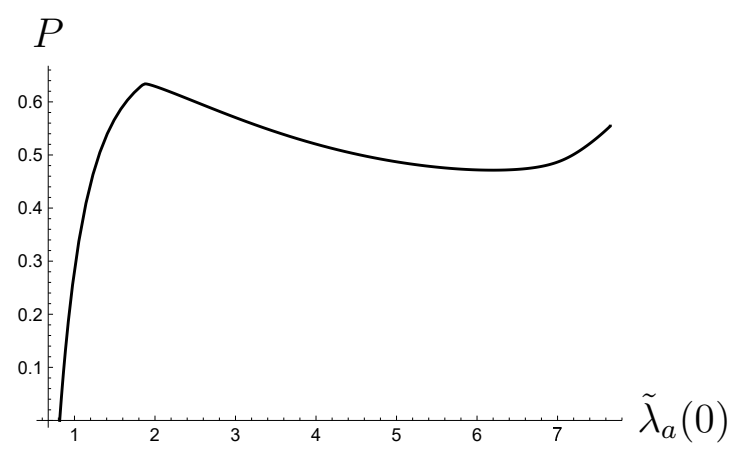

(a)

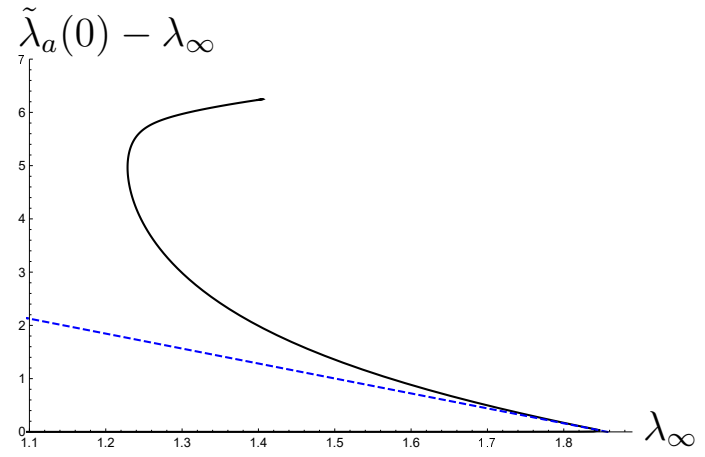

(b)

Figure 5: Amplitude diagrams for a thick-walled tube with $\alpha=0.5$ and $\lambda_{z}$ fixed to be 1.5. The solid lines in both (a) and (b) are numerical simulation results using Abaqus and the dashed line (b) is the weakly nonlinear result given by the full 3D theory.

\subsection{Comparison with Abaqus simulations}

The weakly nonlinear post-buckling solution for $\tilde{\lambda}_{a}(z)$ is given by

$$
\tilde{\lambda}_{a}(z)=\lambda_{\infty}+\frac{u}{A}=\lambda_{\infty}+\varepsilon \frac{1}{A}\left(a+\frac{\Gamma}{a}\right) c_{1}(s)
$$

where $a$ is now the inner radius in the critical configuration given by $a=\lambda_{a \mathrm{cr}} A$. In the above expression we have neglected terms of order $\varepsilon^{2}$. At $z=0$, we have

$$
\begin{aligned}
& \tilde{\lambda}_{a}(0)=\lambda_{\infty}-\frac{1}{A}\left(a+\frac{\Gamma}{a}\right) \frac{3 \varepsilon \lambda_{0} k_{1}}{2 k_{2}}, \\
= & \lambda_{\infty}-\frac{1}{A}\left(a+\frac{\Gamma}{a}\right) \frac{3 k_{1}}{2 k_{2}}\left(\lambda_{\infty}-\lambda_{a \mathrm{cr}}\right) .
\end{aligned}
$$

Thus, the bulge amplitude $\tilde{\lambda}_{a}(0)-\lambda_{\infty}$ is a linear function of $\lambda_{\infty}$. 
For the case when the scaled wall thickness $\alpha$ is no longer small, we compare our weakly nonlinear results with the numerical simulation results obtained with the aid of Abaqus. Our Abaqus implementation is in turn validated by comparing with the membrane theory when $\alpha$ is sufficiently small. Thus, in Figures 2 and 3 we have shown all the three sets of results for a thin-walled tube with $\alpha=0.04$. In each figure the Abaqus simulation results for both the variation of $P$ versus $\tilde{\lambda}_{a}(0)$ and $\tilde{\lambda}_{a}(0)-\lambda_{\infty}$ versus $\lambda_{\infty}$ are shown (as solid lines), where $\tilde{\lambda}_{a}(z)$ denotes the azimuthal stretch on the inner surface in the bulged configuration and $\lambda_{\infty}=\tilde{\lambda}_{a}(\infty)$. For the sake of clarity, in each figure we have only shown the membrane theory result on the left (dashed line) and the weakly nonlinear result on the right (dashed line). In the case of fixed $N$, after reaching its critical value for localized bulging, the pressure decreases monotonically, and approaches, but would never reaches, its propagation value. This value and the associated value of $\tilde{\lambda}_{a}(0)$ can be determined by Maxwell's equal area rule. In contrast, in the case of fixed length, the pressure decreases to a minimum first and then grows slowly, and it is on this ascending branch that the bulge starts to propagate rapidly in the axial direction. It is noted that there is excellent agreement between the membrane theory results and Abaqus simulation results, and in particular both exhibit non-smooth pressure variation at the pressure maximum in the case of fixed axial stretch.

In Figures 2(b), 3(b), 4(b) and 5(b) we have shown the weakly nonlinear results together with the Abaqus simulation results for both the thin-walled tube discussed above and another thick-walled tube with $\alpha=0.5$. It is seen that for all cases the weakly nonlinear result describes the near-critical behaviour correctly. We note that in Figures (3b) and (5b) our weakly nonlinear result corresponds to fixed $\lambda_{z}$ whereas the Abaqus simulations correspond to fixed length. These two end conditions result in different post-bifurcation behavior, but in a sufficiently small neighbourhood of the bifurcation point, the difference is expected to be negligible. This is indeed seen in Figures (3b) and (5b).

\subsection{Effect of increasingly larger and larger $N$ or $\lambda_{z}$}

We recall that inflation can be carried out by fixing either $N$ or $\lambda_{z}$ and localized bulging cannot occur if either of these two values is large enough. In this subsection, we consider a thin-walled tube with $\alpha=0.01$ and investigate the variations of the two coefficients $k_{3}$ and $k_{4}$ with respect to $N$ or $\lambda_{z}$. The results are presented in Tables 3 and 4 where we have also shown the corresponding results obtained under the membrane assumption.

We first discuss the case of fixed $N$ and recall that $N=3.47$ is approximately the maximum value beyond which localized bulging becomes impossible. It is seen in Table 3 that as $N$ is increased towards this maximum value, $k_{3}$ and $k_{4}$ do not change sign, meaning that the bifurcation is always sub-critical and corresponds to bulging. The ratio $k_{3} / k_{4}$ varies between 1.65 and 1.99 which is not significant. However, both $k_{3}$ and $k_{4}$ decrease by several orders of magnitude as the maximum of $N$ is approached. Recall from (3.26) that the 
bulging solution depends on $z$ through $k_{3} s$ so that the smaller the $k_{3}$ is, the slower the bulging solution decays. Thus, for instance, the bulging solution at $N=3.47$ would spread over a domain 30 times as large as the solution at $N=0$. This is consistent with what has been observed in our Abaqus simulations.

We next turn to the case of fixed axial stretch and recall that $\lambda_{z}=3.6$ is approximately the maximum value beyond which localized bulging becomes impossible. It is seen in Table 4 that the manner in which $k_{3}$ and $k_{4}$ behave as $\lambda_{z}$ is increased towards its maximum value is similar to when $N$ is increased towards its maximum value. Thus, the bulging solution would also spread over a larger and larger domain as $\lambda_{z}$ is increased, but there is a major difference between the current and previous cases. It is now observed that $k_{4}$ changes sign as $\lambda_{z}$ is increased beyond the value of 3.5 , whether it is the membrane theory or the $3 \mathrm{D}$ exact theory that is used. This implies that if the tube is first stretched with $\lambda_{z}$ greater than 3.5 and then inflated with the two ends fixed, the first bifurcation will correspond to localized necking, instead of bulging, although the bifurcation is still sub-critical. Similar transition behaviour is also observed for other values of wall thickness. To give this prediction more credence, we have also run a series of numerical simulations with the use of Abaqus, and a typical set of results is given in Figure 6. To produce the profile for necking centered around $z=0$, we have introduced a small imperfection such that the shear modulus for a small middle section is 1.002 times the modulus for the rest of the tube. We also note that neither bulging nor necking is as pronounced as the bulging for much lower values of $\lambda_{z}$. This is a general feature as the maximum value of $N$ or $\lambda_{z}$ is approached, that is when localization has almost become impossible.

Finally, we observe from Tables 3 and 4 that in both cases the membrane theory gives increasingly poorer and poorer predictions for $k_{3}$ and $k_{4}$ as $N$ or $\lambda_{z}$ is increased towards its respective maximum value. A general observation that can be made is that the membrane theory tends to give poor approximations for stiff tubes, as demonstrated in Wang \& $\mathrm{Fu}$ (2018). In the current context, the higher the pre-stretch $\lambda_{z}$, the stiffer the tube becomes. This will be discussed further in the concluding section.

\section{Conclusion}

In this paper we have shown that an amplitude equation describing the shape of a localized bulge/neck can be derived analytically for a tube of arbitrary wall thickness. This equation can be used to determine whether the localization as a bifurcation phenomenon is supercritical or subcritical and whether the bifurcation corresponds to localized bulging or necking. We anticipated that the subcritical nature of the bifurcation was unlikely to 
Table 3: Dependence of $k_{3}$ and $k_{4}$ on $N$ when inflation is carried out with fixed $N$.

\begin{tabular}{ccccccc}
\hline & $k_{3}$ & $k_{3 \mathrm{mem}}$ & $\left|1-k_{3} / k_{3 \mathrm{mem}}\right|$ & $k_{4}$ & $k_{4 \mathrm{mem}}$ & $\left|1-k_{4} / k_{4 \mathrm{mem}}\right|$ \\
\hline$N=0.00$ & -3.21 & -3.22 & $0.287 \%$ & -1.612 & -1.609 & $0.237 \%$ \\
\hline$N=1.00$ & -0.7205 & -0.7103 & $1.44 \%$ & -0.3632 & -0.3551 & $2.27 \%$ \\
\hline$N=2.00$ & -0.1391 & -0.1347 & $3.25 \%$ & -0.07076 & -0.06737 & $5.02 \%$ \\
\hline$N=2.50$ & -0.06020 & -0.0577 & $4.360 \%$ & -0.03091 & -0.02884 & $7.18 \%$ \\
\hline$N=3.00$ & -0.02332 & -0.0218 & $6.89 \%$ & -0.01222 & -0.01091 & $12.0 \%$ \\
\hline$N=3.25$ & -0.01217 & -0.0109 & $11.7 \%$ & -0.006540 & -0.005448 & $20.1 \%$ \\
\hline$N=3.47$ & -0.003299 & -0.000556 & $494 \%$ & -0.001997 & -0.0002775 & $619 \%$ \\
\hline
\end{tabular}

Table 4: Dependence of $k_{3}$ and $k_{4}$ on $\lambda_{z}$ when inflation is carried out with fixed $\lambda_{z}$.

\begin{tabular}{ccccccc}
\hline & $k_{3}$ & $k_{3 \mathrm{mem}}$ & $\left|1-k_{3} / k_{3 \mathrm{mem}}\right|$ & $k_{4}$ & $k_{4 \mathrm{mem}}$ & $\left|1-k_{4} / k_{4 \mathrm{mem}}\right|$ \\
\hline$\lambda_{z}=2.0$ & -0.3131 & -0.3135 & $0.117 \%$ & -0.1597 & -0.1581 & $0.989 \%$ \\
\hline$\lambda_{z}=3.0$ & -0.03475 & -0.03478 & $0.0680 \%$ & -0.01633 & -0.01572 & $3.90 \%$ \\
\hline$\lambda_{z}=3.3$ & -0.01523 & -0.01524 & $0.0554 \%$ & -0.006632 & -0.006179 & $7.34 \%$ \\
\hline$\lambda_{z}=3.4$ & -0.01056 & -0.01057 & $0.0520 \%$ & -0.004249 & -0.003847 & $10.4 \%$ \\
\hline$\lambda_{z}=3.5$ & -0.0064 & -0.006413 & $0.0329 \%$ & -0.002020 & -0.001674 & $20.8 \%$ \\
\hline$\lambda_{z}=3.6$ & -0.002044 & -0.002046 & $0.106 \%$ & 0.0007747 & 0.001048 & $26.1 \%$ \\
\hline
\end{tabular}



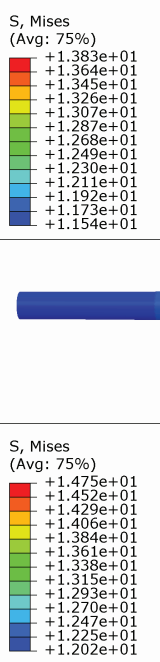

Figure 6: Abaqus simulation results that confirm the theoretical prediction that when $\lambda_{z}=3.5$ bulging occurs (upper figure) whereas when $\lambda_{z}=3.6$ necking occurs (lower figure).

be changed by increased wall thickness, especially for the case of fixed $N$ since the critical pressure corresponds to the pressure maximum in uniform inflation. This is indeed confirmed by our numerical results which describe accurately the behaviour of the bifurcation diagram near the bifurcation point. Additionally, our calculations also reveal that localized bulging may give way to localized necking although it can only occur in the case of fixed axial stretch and in a small interval of the axial stretch for the particular strain-energy function used. This should be noted against the fact that only localized bulging has been reported in previous experimental studies. The validity of the membrane theory in the description of post-bifurcation states is assessed, and it is found that the membrane theory gives increasingly poorer and poorer predictions when the tube is more and more stretched, through either $N$ or $\lambda_{z}$, before inflation is carried out.

Since our earlier study Wang \& Fu (2018) has indicated that the membrane theory is not suitable for finding the critical pressure for fibre-reinforced tubes or tube that exhibit similar stiff behaviour, we decided to also carry out computations for a variety of models for arteries. The most prominent feature associated with arterial models is the existence of a material parameter, $\gamma$ say, that describes the fractional percentage or relative stiffness/extensibility of the reinforcing fibres. It was shown in Wang \& Fu (2018) that for each fixed wall thickness, the contour plot of the bifurcation condition (2.7) would move down as $\gamma$ was increased gradually, disappearing completely when $\gamma$ reaches a critical value. In a similar manner, for each fixed value of $\gamma$, the contour plot of (2.7) would also move down as the wall thickness was increased gradually, disappearing completely when the wall thickness reaches a critical 
value. When the bifurcation condition does not have any solution, the tube will not suffer localized bulging no matter how the tube is inflated (fixed $N$ or fixed length). There also exist intermediate values of $\gamma$ for which localized bulging cannot occur for fixed $N$ but can take place for fixed length. It turns out that all the features described above are also exhibited by the following simple isotropic model that has previously been used by Alhayani et al. (2014):

$$
W=\frac{\mu}{2(1-k+k m)}\left\{(1-k) I_{1}+k \mathrm{e}^{m\left(I_{1}-3\right)}+2 k-3\right\},
$$

where $k$ and $m$ are constants satisfying the conditions $0 \leq k \leq 1$ and $m>0$. It is seen that this is a linear combination of the classical neo-Hookean model and the Demiray model (Demiray, 1996), and the two constants $k$ and $m$ can be viewed as measures of the fractional percentage and stiffness of the reinforcing fibres, respectively. In Figure 7, we have shown the contour plots of the bifurcation condition for the two representative cases $k=0.4, m=$ 0.25 and $k=0.45, m=0.25$, respectively. In each plot we have also shown the curve corresponding to $N=0$, but we note that curves corresponding to other fixed values of $N$ could also be plotted. The right figure corresponding to $k=0.45$ demonstrates the fact that although localized bulging is not possible when $N=0$, it is still possible when it is $\lambda_{z}$ that is fixed instead (the case of fixed length). We note, however, that in the latter case inflation would trace the line $\lambda_{z}=C$ in the $\left(\lambda_{a}, \lambda_{z}\right)$-plane, where $C$ is the fixed value of $\lambda_{z}$, and Euler-type buckling may occur before localized bulging (that is before the line $\lambda_{z}=C$ intersects the bifurcation curve). This has been verified with the aid of Abaqus simulations.

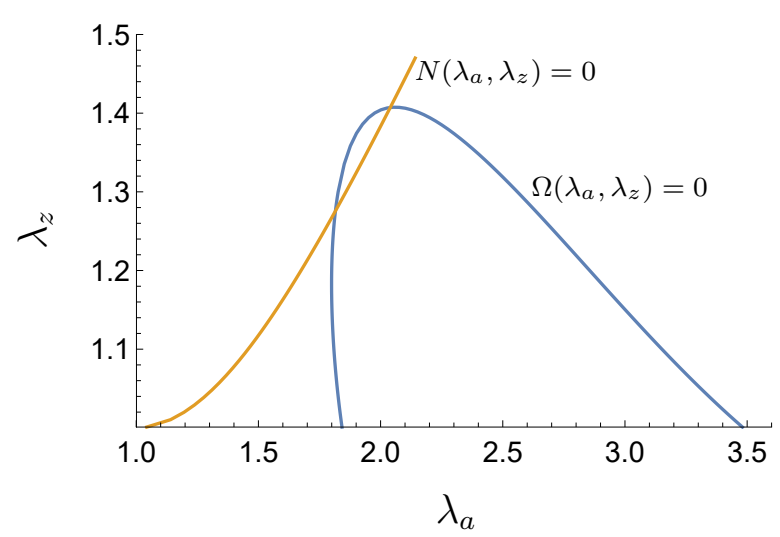

(a)

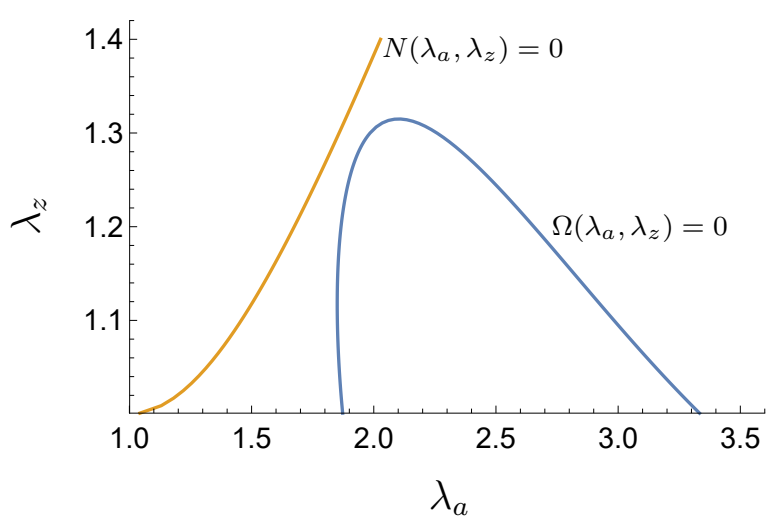

(b)

Figure 7: Contour plots of $\Omega\left(\lambda_{a}, \lambda_{z}\right)=0$ and $N\left(\lambda_{a}, \lambda_{z}\right)=0$ for a tube with $\alpha=0.1$ when the material is given by (5.10). (a) $k=0.4, m=0.25$; (b) $k=0.45, m=0.25$.

In the case when $k=0.4$, Figure 7 (a) shows that localized bulging may occur when either $N=0$ or $\lambda_{z}$ is fixed to be any value between 1.26 and 1.41. Our further calculations show that for all the cases when localized bulging is possible and occurs before Euler-type buckling, the membrane theory gives poor predictions for the coefficients $k_{3}$ and $k_{4}$ even for very thin-walled tubes. Combing this with our earlier observations, we may conclude that 
membrane theory tends to give poor predictions for stiff tubes whether the stiffness is due to fibre reinforcement or excessive stretching in the axial direction.

Acknowledgements

This work was supported by the National Natural Science Foundation of China (Grant Nos 11672202 and 11602163). The Abaqus simulations were carried out on TianHe-1 (A) at the National Supercomputer Center in Tianjin, China.

\section{References}

ABAQUS (2013). ABAQUS Analysis User's Manual, version 6.13.

Alexander, H. (1971). Tensile instability of initially spherical balloons. Int. J. Eng. Sci., 9, $151-160$.

Alhayani, A. A., Giraldo, J. A., Rodríguez1, J., \& Merodio, J. (2013). Computational modelling of bulging of inflated cylindrical shells applicable to aneurysm formation and propagation in arterial wall tissue. Finite Elem. Anal. Des., 73, 20-29.

Alhayani, A. A., Rodríguez, J., \& Merodio, J. (2014). Competition between radial expansion and axial propagation in bulging of inflated cylinders with application to aneurysms propagation in arterial wall tissue. Int. J. Eng. Sci., 85, 74-89.

An, L., Wang, F. F., Cheng, S. B., Lu, T. Q., \& Wang, T. J. (2015). Experimental investigation of the electro-mechanical phase transition in a dielectric elastomer tube. Smart Mater. Struct., 24, 035006.

Benedict, R., Wineman, A., \& Yang, W. H. (1979). The determination of limiting pressure in simultaneous elongation and inflation of nonlinear elastic tubes. Int. J. Solids Struct., $15,241-249$.

Bucchi, A., \& Hearn, G. E. (2013a). Delay or removal of aneurysm formation in the anconda wave energy extraction device. Renewable Energy, 55, 104-119.

Bucchi, A., \& Hearn, G. E. (2013b). Predictions of aneurysm formation in distensible tubes: Part a. theoretical background to alternative approaches. Int. J. Mech. Sci., 71, 1-20.

Cai, Z. X., \& Fu, Y. B. (1999). On the imperfection sensitivity of a coated elastic half-space. Proc. Roy. Soc. Lond. A, 455, 3285-3309.

Cao, Y. P., \& Hutchinson, J. W. (2012). Wrinkling phenomena in neo-hookean film/substrate bilayers. ASME J. Appl. Mech., 79, 031019.

Chakrabarti, A., Mora, S., Richard, F., Phou, T., Fromental, J.-M., Pomeau, Y., \& Audoly, B. (2018). Selection of hexagonal buckling patterns by the elastic Rayleigh-Taylor instability. J Mech. Phys. Solids, 121, 234-257. 
Chater, E., \& Hutchinson, J. W. (1991). On the propagation of bulges and buckles. ASME J. Appl. Mech., 51, 269-277.

Chen, Y. C. (1997). Stability and bifurcation of finite deformations of elastic cylindrical membranes - part i. stability analysis. Int. J. Solids Struct., 34, 1735-1749.

Chen, Y. C., \& Haughton, D. M. (2003). Stability and bifurcation of inflation of elastic cylinders. Proc. R. Soc. Lond. A, 459, 137-156.

Demiray, H. (1996). Solitary waves in initially stressed thin elastic tubes. Int. J. Non-Linear Mech., 32, 1165-1176.

Demirkoparan, H., \& Merodio, J. (2017a). Bulging bifurcation of inflated circular cylinders of doubly fiber-reinforced hyperelastic material under axial loading and swelling. Math. Mech. Solids, 22, 666-682.

Demirkoparan, H., \& Merodio, J. (2017b). Swelling and axial propagation of bulging with application to aneurysm propagation in arteries. Math. Mech. Solids, doi: 10.1007/s10665014-9771-9.

Fu, Y. B. (1995). Resonant-triad instability of a pre-stressed elastic plate. J. Elasticity, 41, $13-37$.

Fu, Y. B. (2001). Nonlinear stability analysis. In Nonlinear elasticity: theory and applications (eds YB Fu, RW Ogden). Cambridge University Press, Cambridge.

Fu, Y. B., Dorfmann, L., \& Xie, Y. X. (2018). Localized necking of a dielectric membrane. Extreme Mech. Lett., 21, 44-48.

Fu, Y. B., \& Ilíchev, A. T. (2010). Solitary waves in fluid-filled elastic tubes: existence, persistence, and the role of axial displacement. IMA J. Appl. Math., 75, 257-268.

Fu, Y. B., \& Ilíchev, A. T. (2015). Localized standing waves in a hyperelastic membrane tube and their stabilization by a mean flow. Maths Mech. Solids, 20, 1198-1214.

Fu, Y. B., Liu, J. L., \& Francisco, G. S. (2016). Localized bulging in an inflated cylindrical tube of arbitrary thickness - the effect of bending stiffness. J. Mech. Phys. Solids, 90, $45-60$.

Fu, Y. B., \& Ogden, R. W. (1999). Nonlinear stability analysis of pre-stressed elastic bodies. Continuum Mech. Thermodyn., 11, 141-172.

Fu, Y. B., Pearce, S. P., \& Liu, K.-K. (2008). Post-bifurcation analysis of a thin-walled hyperelastic tube under inflation. Int. J. Non-linear Mech., 43, 697-706. 
Fu, Y. B., \& Rogerson, G. A. (1994). A nonlinear analysis of instability of a pre-stressed incompressible elastic platet. Proc. R. Soc. Lond. A, 446, 233-254.

Fu, Y. B., Rogerson, G. A., \& Zhang, Y. T. (2012). Initiation of aneurysms as a mechanical bifurcation phenomenon. Int. J. Non-linear Mech., 47, 179-184.

Fu, Y. B., \& Xie, Y. X. (2010). Stability of localized bulging in inflated membrane tubes under volume control. Int. J. Eng. Sci., 48, 1242-1252.

Fu, Y. B., \& Xie, Y. X. (2012). Effects of imperfections on localized bulging in inflated membrane tubes. Phil. Trans. R. Soc., A 370, 1896-1911.

Gent, A. (1996). A new constitutive relation for rubber. Rubber Chem. Technol., 69, 59-61.

Goncalves, P. B., Pamplona, D. C., \& Lopes, S. R. X. (2008). Finite deformations of an initially stressed cylindrical shell under internal pressure. Int. J. Mech. Sci., 50, 92-103.

Green, A. E., \& Zerna, W. (1954). Theoretical Elasticity. The Clarendon Press, Oxford.

Haughton, D. M., \& Ogden, R. W. (1979a). Bifurcation of inflated circular cylinders of elastic material under axial loading i. membrane theory for thin-walled tubes. J. Mech. Phy. Solids, 27, 179-212.

Haughton, D. M., \& Ogden, R. W. (1979b). Bifurcation of inflated circular cylinders of elastic material under axial loading ii. exact theory for thick-walled tubes. J. Mech. Phy. Solids, 27, 489-512.

van der Heijden, A. M. A. (2009). WT Koiter's elastic stability of solids and structures. Cambridge University Press, Cambridge.

Horny, L., Netusil, M., \& Horak, Z. (2015). Limit point instability in pressurization of anisotropic finitely extensible hyperelastic thin walled tube. Int. J. Non-Linear Mec., 77, $107-114$.

Hutchinson, J. W. (2012). The role of nonlinear substrate elasticity in the wrinkling of thin films. Phil. Trans. R. Soc. A, 371, 20120422.

Hutchinson, J. W., \& Koiter, W. T. (1972). Postbuckling theory. Appl. Mech. Rev., 23, $1353 \mathrm{C} 1365$.

Ilíchev, A. T., \& Fu, Y. B. (2012). Stability of aneurysm solutions in a fluid-filled elastic membrane tube. Acta Mechanica Sinica, 28, 1209-1218.

Ilíchev, A. T., \& Fu, Y. B. (2014). Stability of an inflated hyperelastic membrane tube with localized wall thinning. Int. J. Eng. Sci., 80, 53-61. 
Jin, L. S., Liu, Y., \& Cai, Z. X. (2019). Post-buckling analysis on growing tubular tissues: A semi-analytical approach and imperfection sensitivity. Int. J. Solids Struct., 162, 121-134.

Kanner, L. M., \& Horgan, C. O. (2007). Elastic instabilities for strain-stiffening rubber-like spherical and cylindrical thin shells under inflation. Int. J. Non-linear Mech., 42, 204-215.

Kyriakides, S., \& Chang, Y.-C. (1990). On the inflation of a long elastic tube in the presence of axial load. Int. J. Solids Struct., 26, 975-991.

Kyriakides, S., \& Chang, Y.-C. (1991). The initiation and propagation of a localized instability in an inflated elastic tube. Int. J. Solids Struct., 27, 1085-1111.

Lestringant, C., \& Audoly, B. (2018). A diffuse interface model for the analysis of propagating bulges in cylindrical balloons. Proc. Roy. Soc. A, 474, 20180333.

Liu, Y., Ye, Y., Althoubaiti, A., \& Xie, Y. X. (2019). Prevention of localized bulging in an inflated bilayer tube. Int. J. Mech. Sci., 153-154, 359-368.

Lu, T. Q., An, L., Li, J. G., Yuan, C., \& Wang, T. J. (2015). Electro-mechanical coupling bifurcation and bulging propagation in a cylindrical dielectric elastomer tube. J. Mech. Phy. Solids, 85, 160-175.

Merodio, J., \& Haughton, D. (2010). Bifurcation of thick-walled cylindrical shells and the mechanical response of arterial tissue affected by Marfans syndrome. Mech. Res. Comm., 37, 1-6.

Ogden, R. W. (1984). Non-linear Elastic Deformations. Ellis Horwood, New York.

Pamplona, D. C., Goncalves, P. B., \& Lopes, S. R. X. (2006). Finite deformations of cylindrical membrane under internal pressure. Int. J. Mech. Sci., 48, 683-696.

Pearce, S. P., \& Fu, Y. B. (2010). Characterisation and stability of localised bulging/necking in inflated membrane tubes. IMA J. Appl. Math., 75, 581-602.

Potier-Ferry, M. (1987). Foundations of elastic post-buckling theory, in Buckling and postbuckling (ed. J. Arbocz et al), Lecture Notes in Physics Vol. 288. Springer, Berlin.

Ren, J. S. (2007). Inflation of an artery leading to aneurysm formation and rupture. Mol. Cell Biomech., 4, 55-66.

Ren, J. S., Zou, J. W., \& Yuan, X. G. (2011). Instability analysis in pressurized three-layered fiber-reinforced anisotropic rubber tubes in torsion. Int. J. Eng. Sci., 49, 342-353.

Richard, F., Chakrabarti, A., Audoly, B., Pomeau, Y., \& Mora, S. (2018). Buckling of a spinning elastic cylinder: linear, weakly nonlinear and post-buckling analyses. Proc. $R$. Soc. Lond. A, 474, 20180242. 
Rodriguez, J., \& Merodio, J. (2011). A new derivation of the bifurcation conditions of inflated cylindrical membranes of elastic material under axial loading. Application to aneurysm formation. Mech. Res. Comm., 38, 203-210.

Sawyers, K. N., \& Rivlin, R. S. (1982). Stability of a thick elastic plate under thrust. J. Elast., 12, 101-125.

Shi, J., \& Moita, G. F. (1996). The post-critical analysis of axisymmetric hyper-elastic membranes by the finite element method. Comput. Methods Appl. Mech. Engrg, 135, $265-281$.

Shield, R. T. (1972). On the stability of finitely deformed elastic membranes; part ii: stability of inflated cylindrical and spherical membranes. ZAMP, 23, 16-34.

Takla, M. (2018). Bifurcation of elastic-plastic thick-walled cylindrical structures. Int. J. Mech. Sci., 141, 303-315.

Triantafyllidis, N., Scherzinger, W. M., \& Huang, H.-J. (2007). Post-bifurcation equilibria in the plane-strain test of a hyperelastic rectangular block. Int. J. Solids Struct., 44 , 3700-3719.

Varatharajan, N., \& DasGupta, A. (2017). Study of bifurcation in a pressurized hyperelastic membrane tube enclosed by a soft substrate. Int. J. Non-linear Mech., 95, 233-241.

Wang, F. F., Chao, Y., Lu, T. Q., \& Wang, T. J. (2017a). Anomalous bulging behaviors of a dielectric elastomer balloon under internal pressure and electric actuation. J. Mech. Phys. Solids, 102, 1-16.

Wang, J., Althobaiti, A., \& Fu, Y. B. (2017b). Localized bulging of rotating elastic cylinders and tubes. J. Mech. Mater. Struct., 12, 545-561.

Wang, J., \& Fu, Y. B. (2018). Effect of double-fibre reinforcement on localized bulging of an inflated cylindrical tube of arbitrary thickness. J. Eng. Math., 109, 21-30.

Wang, S. B., Guo, G. M., Zhou, L., Li, L. A., \& Fu, Y. B. (2019). An experimental study of localized bulging in inflated cylindrical tubes guided by newly emerged analytical results. J. Mech. Phy. Solids, 124, 536-554.

Wineman, A. S. (2015). Determining the time of bulge formation in an elastomeric tube as it inflates, elongates and alters chemorheologically. Math. Mech. Solids, 20, 9-24.

Wineman, A. S. (2017). Bulge initiation in tubes of time-dependent materials. Math. Mech. Solids, 22, 636-648. 
Wolfram Research Inc (2019). Mathematica: version 12. Wolfram Research Inc, Champaign, IL..

Ye, Y., Liu, Y., Althobaiti, A., \& Xie, Y. X. (2019). Localized bulging in an inflated bilayer tube of arbitrary thickness: effects of the stiffness ratio and constitutive model. Int. J. Solids Struct., 176-177, 173-184.

Yin, W.-L. (1977). Non-uniform inflation of a cylindrical elastic membrane and direct determination of the strain energy function. J. Elast., 7, 265-282. 